\title{
INDICADORES EN EL SERVICIO EN LAS PEQUEÑAS Y MEDIANAS EMPRESAS RESTAURANTERAS EN MÉXICO: UN ABORDAJE COMPARATIVO ${ }^{1}$
}

\author{
Aida Salazar Alcaraz* \\ https://orcid.org/0000-0002-6272-5141 \\ Lisandro Alvarado-Peña* \\ https://orcid.org/0000-0001-5097-811X \\ Luis Vega Osuna* \\ https://orcid.org/0000-0003-3844-2245 \\ Flor Salaiza Lizárraga* \\ https://orcid.org/0000-0003-3864-0575
}

RECIBIDO: Enero 2020 / ACEPTADO: Junio 2020 / PUBLICADO: Septiembre 2020

Como citar: Salazar Alcaraz, Aida; Alvarado-Peña, Lisandro; Vega Osuna, Luis; Salaiza Lizárraga, Flor. (2020) Indicadores en el servicio en las pequeñas y medianas empresas restauranteras en méxico: un abordaje comparativo. Telos: revista de Estudios Interdisciplinarios en Ciencias Sociales, 22 (3), Venezuela. (Pp.551-566).

DOI: www.doi.org/10.36390/telos223.06

\section{RESUMEN}

Los restaurantes de la región se caracterizan por un servicio y oferta gastronómica que determinan el nivel de preferencia de los clientes, por lo que este artículo tiene como objetivo analizar la opinión de los consumidores, a través de una comparación del servicio de cocina regional; en este proceso se analizan los restaurantes Pascola y Casa de Peraza, instalados en la ciudad de Culiacán Sinaloa, México. La metodología corresponde a la investigación descriptiva transversal, con una encuesta y muestra por conveniencia de 134 clientes. El instrumento contiene 20 indicadores desde la perspectiva teórica de la

\footnotetext{
1 Este artículo forma parte de una investigación amplia que se registra en el Instituto de Investigaciones de la Red Académica Internacional de Estudios Organizacionales en América Latina, el Caribe e Iberoamérica (Red REOALCEI) de forma conjunta con el Cuerpo Académico Desarrollo Organizacional UDO-CA3.

* Doctorado en Tecnología Educativa. Maestra en Administración y Licenciatura en Administración. Profesora de la Universidad Autónoma de Occidente, Culiacán, México. Integrante del Cuerpo Académico en el nivel de consolidación "Desarrollo Organizacional, correo-e: aidasalcaraz@gmail.com

* Doctorado en Ciencias Sociales Mención: Gerencia. Maestro en Gerencia Pública. Diploma de Estudios Avanzados (DEA) dentro del Doctorado en Administración de Empresas de la Universidad Politécnica de Madrid(UPM)-Madrid, España. Licenciatura en Administración de Empresas. Profesor Investigador de la Universidad de Zulia-Escuela de Economía-FCES. Investigador del Instituto de Investigaciones de la Red Académica Internacional "Estudios Organizacionales en América Latina, el Caribe e Iberoamérica" (REOALCEI). Correo-e: lisandro.investigacion.reoalcei@gmail.com

* Doctorado en Ciencias Administrativas inscrito en el PNPC-Conacyt. Maestro en Comercio Exterior y la Licenciatura en Comercio Internacional. Profesor investigador de la Universidad Autónoma de Occidente, miembro del núcleo académico básico del Doctorado en Ciencias Administrativas. Integrante del Cuerpo Académico en el nivel de consolidación "Desarrollo Organizacional". Investigador del Instituto de Investigaciones de la Red Académica Internacional "Estudios Organizacionales en América Latina, el Caribe e Iberoamérica" (REOALCEI). Correo electrónico luisalfredovega922@gmail.com

* Doctorado en Administración. Maestra en Negocios Internacionales y en Ciencias de la Administración y la Licenciatura en Sistemas de Computación Administrativa. Profesora del Tecnológico Nacional de México campus Instituto Tecnológico de Culiacán, miembro del núcleo académico básico del posgrado en la Universidad de San Miguel. Correo electrónico fsalaiza@itculiacan.edu.mx
} 
Indicadores en el servicio en las pequeñas y medianas empresas restauranteras en México: un abordaje comparativo

mercadotecnia de servicio y el modelo de las 4C de Lauterborn descritas por Kotler y Keller (2006), lo que orienta el trabajo de campo y análisis en cuatro dimensiones: consumidor, costo, conveniencia y comunicación. Los antecedentes de investigación corresponden a Hernández Rojas y Dancausa Millán (2018) y Villanueva Cueva (2017); así como en las cuatro dimensiones: Lerma (2010), Kotler y Armstrong (2003), Tschohl y Franzmeier (1994), Cuevas (2004), y Hernández Garnica y Maubert Viveros (2009). El concepto de servicio de restaurante, tiene como principal referente teórico a Villegas Becerril (2014). Se concluye que es importante que los restaurantes ofrezcan un buen servicio y calidad a los comensales; que realicen con regularidad sondeos de opinión para atender las necesidades y mejoren el servicio; asimismo ofrezcan promociones de cliente frecuente; bufet los fines de semana y consideren la protección del consumidor además de la seguridad del establecimiento.

Palabras clave: preparación de alimentos, servicio al cliente, consumidor, turismo, empresa privada.

\title{
Indicators in the service in small and medium-sized restaurant companies in Mexico: a comparative approach
}

\begin{abstract}
The restaurants of the region are characterized for the service and the gastronomic offer that determines the level of preferences of its customers, the object of this article is analyze the opinion of the customers, in base of a comparison for the service in a regional kitchen; in this process we analyze the restaurants Pascola and Casa de Peraza, that are placed in the city of Culiacan Sinaloa, Mexico. The methodology corresponds to a cross descriptive research, with a poll and simple for convenience from 134 clients. The instrument contains 20 indicators from the theorical perspective from the marketing of service and the $4 \mathrm{C}$ model from Robert Lauterborn, described by Kotler and Keller (2006), and guides the field work and analysis in four dimensions: consumer, cost, convenience and communication. The background of the investigation corresponds to Hernandez Rojas and Dancausa Millan (2018), and Villanueva Cueva (2017); and also, in four dimensions: Lerma Kirchner (2010), Kotler and Armstrong (2003), Tschohl and Franzmeier (1994), Cuevas (2004) and, Hernandez Garnica and Maubert Viveros (2009). The concept of the service of the restaurant, have the principal theorical reference to Villegas Becerril (2014). It concludes that it's important for the restaurants offer a great service and quality to his dinners; realize with regularity opinion polls for attend the needs and improve the service; likewise, offer promotions for being a frequent client, buffets during weekends and; consider the protection of the customer and also the security of the place.
\end{abstract}

Keywords: foodservice, customer service, consumer, tourism, private business

\section{Introducción}

Esta investigación analiza las características que presenta la oferta gastronómica del menú de desayuno regional, en dos empresas pequeñas de servicio de restaurante instaladas en la ciudad de Culiacán Sinaloa, México. La elección del tipo de restaurante y el concepto de alimentos preparados para su consumo, tiene en cuenta el total de establecimientos formales con un alto crecimiento en la ciudad en los años recientes; lo cual se confirma con datos del sector dados a conocer por la Secretaría de Turismo del Gobierno Federal con base en el Anuario Estadístico y Geográfico de Sinaloa (INEGI, 2017). Las próximas estadísticas del sector se actualizarán con la publicación de resultados de los Censos Económicos 2019, referidos al 
2018, sobre establecimientos productores de bienes, comercializadores de mercancías y prestadores de servicios.

Las estadísticas del sector divulgadas por el INEGI (2017),en el apartado de establecimientos de preparación y servicio de alimentos y bebidas con categoría turística y conexas, para el estado de Sinaloa indican un total de 2,424 lugares donde se preparan y se da servicio de alimentos y de bebidas, de los cuales 1,619 son restaurantes (el resto incluye a establecimientos como cafeterías, neverías, refresquerías, centros nocturnos y discotecas, bares y cantinas) localizados en los 18 municipios que integran la entidad federativa, donde la capital del estado es Culiacán. En orden descendente, la cantidad de restaurantes en los principales municipios son: Mazatlán (531), Culiacán (420), Ahome (251) y Guasave (83). Las últimas cuatro posiciones las ocupan Angostura (9), Elota (9), Badiraguato (7), Choix (6) y Sinaloa municipio (4).

La investigación se delimita a los establecimientos especializados en platillos de desayuno regional, para analizar las características del servicio y contribuir con datos relevantes sobre la opinión que expresan los clientes, en torno a los restaurantes típicos de la región. El interés se centra en responder la interrogante: ¿Qué opinan los clientes acerca del servicio de restaurante regional en cuanto a la atención de necesidades, el costo, conveniencia y comunicación? Por lo tanto, se establece como objeto de estudio el servicio de restaurante regional donde las unidades de análisis son dos establecimientos ubicados en la ciudad de Culiacán, en el noroeste de México. Se formula como supuesto de la investigación el siguiente: la opinión es favorable al servicio del restaurante regional con una alta presencia de la comida a base de recetas e ingredientes tradicionales; así mismo, los precios y la ubicación son satisfactorios para la experiencia de consumo en el restaurante.

De las investigaciones seleccionadas como antecedentes, en la primera se aborda el tema del turismo gastronómico, en la que cabe destacar los aportes de Hernández Rojas y Dancausa Millán (2018) quienes a partir del objetivo general: indagar y generar conocimiento desde el punto de vista turístico de los platillos tradicionales en la región de Córdoba, España, concluyeron que los platos propios de los restaurantes de la región son percibidos como singulares y tienen una alta valoración por los empresarios y los turistas gastronómicos. Una segunda investigación considera las $4 \mathrm{C}$ de la mercadotecnia del servicio (el cliente 0 consumidor, el costo-beneficio, la conveniencia y la comunicación), encabezada por Villanueva Cueva (2017) en la ciudad de Trujillo, Perú; en la que una importante contribución metodológica es la aplicación de un cuestionario que sintetiza la información y el instrumento para un estudio detallado del cliente, en los establecimientos de restauración del sector turístico.

La presente investigación descriptiva transversal corresponde a resultados preliminares obtenidos en el primer semestre del año 2019, a partir del trabajo de campo estructurado con las dimensiones e indicadores del servicio, por medio de una encuesta a los comensales en la interpretación de resultados se recurre al modelo de las 4C de Lauterborn (1990) como perspectiva teórica. Por lo que se plantea como objetivo de la investigación: Analizar la opinión de los consumidores, a través de una comparación del servicio de dos restaurantes de cocina regional.

A partir del total de restaurantes establecidos en la ciudad de Culiacán, se define una serie de criterios para seleccionar las unidades de estudio. En los restaurantes potenciales se observa: la ubicación del establecimiento (en el área urbana), los insumos utilizados en la 
Indicadores en el servicio en las pequeñas y medianas empresas restauranteras en México: un abordaje comparativo

preparación de alimentos (los desayunos sinaloenses se caracterizan por el uso de ingredientes del campo y las recetas rurales), la demanda diaria de clientes (el flujo suficiente para realizar el trabajo de campo en un máximo de dos a tres visitas, considerada la posibilidad de rechazo de los comensales), el concepto cultural o tradicional de las instalaciones (un restaurante con identidad local o regional), negocio formal(con registro de contribuyente y licencias según el giro), presencia local mínima de tres años (para confirmar su estabilidad y permanencia en el sector), que cuente con una matriz y sucursal (lo cual se considera un factor de crecimiento), interés del propietario al momento del contacto por conocer el proceso y resultados de la investigación; es decir, que haya condiciones, facilidades y el compromiso para acceder al establecimiento, abordar a los clientes y realizar la encuesta por el equipo de investigación.

Con estos criterios, los candidatos localizados en el centro histórico 0 área conurbada con los que se entra en contacto son 17 restaurantes, a quienes se exponen las generalidades del proyecto; de éstos, quienes deciden apoyar la investigación y posteriormente analizar los resultados son los respectivos propietarios de los restaurantes Pascola y Casa de Peraza. Al rechazar la encuesta los dueños exponen que a los comensales no hay que abordarlos con un fin diferente al consumo de alimentos, como también tienen reservas sobre el contenido mismo del instrumento (el principal motivo es que un resultado adverso los pone en evidencia y no conviene al negocio si la información se divulga). La muestra de comensales es de 134 en total y 67 por cada restaurante. El cuestionario contiene respuestas de opción múltiple, en formato impreso y es auto administrado por los clientes al término del consumo.

La estructura que integra este artículo se compone en primer lugar de la revisión de la literatura con base en dos aspectos teóricos fundamentales para la investigación: el modelo de servicio de las 4C de Lauterborn (analizado por Kotler y Keller, 2006) y el servicio de restaurante. En segundo lugar, se presenta el método de investigación, materiales e instrumento utilizado. Posteriormente se exponen los resultados de la investigación, para luego interpretarlos. Por último, se incluyen las conclusiones y la bibliografía consultada.

\section{Bases teórico conceptuales}

La evolución que ha tenido el concepto de mercadotecnia a través del tiempo ha hecho posible que las compañías pasen del modelo tradicional de la mezcla de marketing (precio, producto, plaza y promoción) propuesto por McCarthy (1960) cuyo enfoque está basado en satisfacer al cliente en el corto plazo lo que se le conoce como marketing transaccional a la mercadotecnia relacional que consiste en generar valor a los clientes y a las relaciones trascendiendo a mediano y largo plazo (De Sá, 2012).

El Concepto de mezcla de mercadotecnia o marketing mix conocido como las 4P (Precio, Producto, Plaza y Promoción) ha estado evolucionando del modelo propuesto por McCarthy (1960) al modelo de las 4C (consumidor, costo, conveniencia y comunicación) propuesto por Lauterborn (1990) donde este último está más orientado a las necesidades que presenta el cliente a diferencia de las 4P que se enfoca más en las habilidades del vendedor; dándole importancia a la forma de generar ingresos a través de las ventas (De Sá, 2012 y Londhe, 2014).

La fundamentación teórica establecida para esta investigación se organiza en dos apartados: el modelo de las 4C, La calidad del producto, del servicio y del entorno físico, el servicio de restaurante y la importancia del análisis del modelo de las $4 \mathrm{C}$ para identificar las 
necesidades de los clientes. Son autores destacados en el área de la mercadotecnia de servicio: Lerma (2010), Kotler y Keller (2006), Kotler y Armstrong (2003), Tschohl y Franzmeier (1994), Cuevas (2004) y, Hernández Garnica y Maubert Viveros (2009). En el servicio de restaurante, los aportes teóricos provienen de: Villegas (2014), García Ortiz et al.,(2011), López Alonso et al., (2017) y Díaz y León (2014).

\section{El modelo de las $4 \mathrm{C}$ de Lauterborn}

El modelo fue diseñado en el año de 1990 por Lauterborn (1990) quien identifica cuatro elementos que están presentes en la mercadotecnia de servicio: consumidor, costo, conveniencia y comunicación. Para Kotler y Keller (2006, p.20) "las empresas que puedan satisfacer las necesidades de los consumidores de forma económica y conveniente, y con una comunicación eficaz, serán las que más triunfen en el futuro". Por otra parte, Lerma (2010) define la mezcla de mercadotecnia ampliada por las 4C:

El valor de uso del producto para el cliente es su utilidad y capacidad de satisfacer las necesidades y los deseos del consumidor. El costo al cliente es el precio total que tiene que pagar el comprador por el producto. Esto incluye el precio del producto más los gastos y costos adicionales que supone su adquisición como transporte, seguros, instalación, póliza de servicio, etcétera. La conveniencia para el comprador es el equivalente de la facilidad y la comodidad para adquirir el producto, basado en los puntos de compra y en los canales de distribución. La comunicación es un diálogo bidireccional entre la empresa oferente y los clientes o posibles clientes a fin de proveer el producto o suministrar el servicio. Esto es hacer promoción con comunicación en ambos sentidos (p. 19-20)

Otras ideas que establecen los expertos permiten complementar la conceptualización de cada uno de los elementos del modelo de las $4 \mathrm{C}$ de Lauterborn: consumidor, costo, conveniencia y comunicación.

a. Consumidor: para Kotler y Armstrong (2003) los clientes en los mercados de consumo consisten en individuos y hogares que compran bienes y servicios para su consumo personal. En términos de Tschohl y Franzmeier (1994)es importante conocer los deseos y necesidades de los clientes 0 consumidores antes de pretender venderles cualquier tipo de servicio o producto. Si usted no los conoce, sencillamente está adivinando.

b. Costo: en el caso de los restaurantes, Cuevas (2004, p.36) expone que "cuantificar el costo en este tipo de empresas es considerando el valor de los materiales usados (ingredientes), ya que al cliente no le interesan los procesos (...) sino la calidad, presentación, cantidad y sabor del platillo o bebida". C. Conveniencia: la definición de Hernández Garnica y Maubert Viveros $(2009$, p.20) relaciona la conveniencia con "establecer una ruta específica para la distribución de los productos en los lugares que sean los más convenientes para todos los que intervienen en el proceso de compra". El productor debe considerar junto a la conveniencia lo accesible que resulte el establecimiento y consumo.

d.Comunicación: en la opinión de Hernández Garnica y Maubert Viveros $(2009$, p.20) la comunicación busca "establecer una relación permanente entre el consumidor y el productor, se trata de que haya un mensaje que reciba el consumidor pero que tenga la posibilidad de darle a conocer al productor lo que piensa de su oferta completa".

El modelo de Lauterborn y la conceptualización teórica reunida, facilita tomar en cuenta, entre otras, las siguientes consideraciones: (a) la mercadotecnia se centra en el estudio de necesidades del consumidor, no en el producto; (b) se requiere estudiar los costos asociados, tiempo y esfuerzo, 
Indicadores en el servicio en las pequeñas y medianas empresas restauranteras en México: un abordaje comparativo

no cómo lograr el menor precio; (c) el consumidor adquiere el bien o servicio en el lugar más accesible $y(d)$ los esfuerzos en la mercadotecnia actual, empiezan por informar a los consumidores sobre nuevos productos o servicios; lo que obliga a pedir su opinión, con la posibilidad de construir y mantener actualizada una base de datos confiable de clientes.

\section{La calidad del producto, del servicio y del entorno físico.}

De acuerdo con Rodríguez-López et al., (2020) al haber analizado varios autores la experiencia que se vive en un restaurante está determinada por la calidad del producto, del servicio y del entorno físico. Cada uno de estos factores juega un rol determinante en la experiencia gastronómica que se vive en el restaurante al momento de que el cliente realice su consumo.

Asimismo, Rodríguez-López et al., (2020) considera seis atributos a la calidad de los alimentos: (1) la frescura, (2) el tamaño del plato, (3) la variedad del menú, (4) la presentación, (5) el sabor y (6) la temperatura. en calidad de servicio se toman en cuenta cinco dimensiones: Elementos que son tangibles (Disponibilidad de utensilios, mesas, sillas, etc.), fiabilidad del restaurante, capacidad de respuesta, seguridad y empatía.

En el entorno Físico Rodríguez-López et al., (2020) que el ambiente físico de un restaurante tiene 3 dimensiones: (1) ambiente: ruido, aromas, iluminación, música, (2) espacio: muebles y (3) símbolos: decoración.

\section{El servicio de restaurante}

Con base en las tendencias y la oferta gastronómica actual se dispone de diversas clasificaciones en torno al servicio de restaurantes. Los especialistas de la gastronomía en general, clasifican a los restaurantes según el tipo de comida en: convencional, casas de comida, de lujo, étnico-regional, temático y monoproducto; la principal característica del restaurante étnico-regional es la oferta gastronómica típica de determinadas regiones o países. En este sentido, Villegas Becerril (2014) destaca en el restaurante regional, el encanto de una cocina que define un espacio concreto, que produce ciertos alimentos diferentes de gran calidad y es uno de los mejores recursos del turismo gastronómico.

Por otra parte, con el criterio del tamaño de empresa, se puede encontrar la presencia de negocios de restaurante tradicional de único establecimiento que se caracteriza como microempresa con baja tecnificación y gestión interna a prueba y error; en otros términos "poca implantación de los avances técnicos tanto en materias primas como en equipos. En su oferta priman las elaboraciones tradicionales a base de productos frescos y el servicio directo, personal y familiar" (García Ortizet al., 2011, p. 13). Pertenecen al sector terciario o de servicios y a su vez en el ámbito de la restauración comercial; es decir, son empresas "que se dedican a prestar servicios de comidas y bebidas en una situación de libre mercado, oferta variada, abierta al público en general e independiente de cualquier actividad" (p. 6). Otros tipos de empresas con sistemas y procedimientos más complejos se organizan con una gestión empresarial en la forma de sucursales, cadenas y franquicias.

El criterio de la oferta diferenciada de clientes es utilizada para identificar: el restaurante convencional (gourmet), restaurante de lujo (alta gama), restaurante regional (platos típicos), restaurante internacional (típico de un país), restaurante monoproducto (producto base), restaurante temático y restaurante de especialidades (López Alonso et al.,2017).

La oferta global de un restaurante según Díaz Paniagua y León Sánchez (2014) la componen el servicio básico (el negocio en el que opera), los servicios periféricos (necesarios para configurar el servicio básico y el cliente los puede usar) y los servicios complementarios (para da un valor agregado al servicio). 
Las interiores tipologías son útiles en la selección de las unidades de estudio, debido a que la oferta gastronómica de los restaurantes es muy variada; y para delimitar la investigación, el criterio del tipo de cocina y servicio de Villegas Becerril (2014) establece los parámetros que caracteriza a los restaurantes regionales: la gastronomía típica. Este tipo de restaurantes típicos son percibidos por los grupos de interés o stakeholders como un apoyo a la comunidad mediante la promoción del turismo con la oferta de comida típica.

\section{Importancia del Análisis del Modelo de las 4C.}

Cabe mencionar que es relevante identificar las opiniones de los clientes con respecto a la calidad en el producto, servicio y del entorno físico que presenta los restaurantes y el modelo de las $4 \mathrm{C}$ en su esencia es crear relaciones de valor con sus clientes buscando la lealtad de los mismos en el mediano y largo plazo.

En la figura 1 se muestran como los factores: el entorno, la competencia, los cambios en el marco jurídico de los gobiernos y la aparición de nueva tecnología influyen en la forma de pensar de los comensales (González et al., 2019). Es por esta razón de que es primordial analizar mediante el modelo de las $4 \mathrm{C}$ (consumidor, costo, conveniencia y comunicación)cómo han evolucionado los comentarios que tiene los clientes de estos restaurantes para identificar que estrategias han sido adecuadas con las necesidades que presentan y que aspectos necesitan mejorar con el objeto de generar valor en los productos, el servicio y el ambiente físico que presentan estos restaurantes generando en el administrador un pensamiento estratégico que se traduce en el desarrollo de estrategias en el presente y en el futuro apegados al marketing relacional (Rodríguez-López et al., 2020, García, 2010 y De Sá, 2012).

Figura 1. Síntesis del Proceso Creación del valor en el cliente a través del modelo de las $4 \mathrm{C}$ propuesto por Lauterborn.

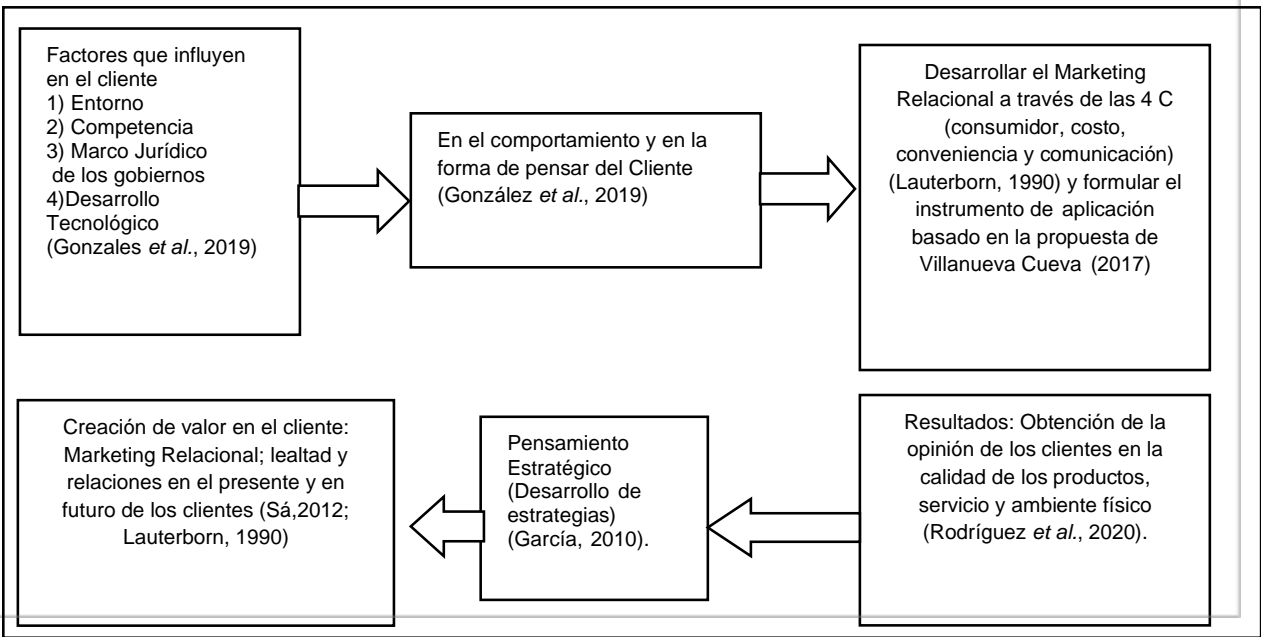

Fuente: Elaboración propia a partir de los constructos de Lauterborn (1990); García (2010); De Sá (2012); Villanueva Cueva (2017); González et al., (2019) y Rodríguez-López et al., (2020). 
Indicadores en el servicio en las pequeñas y medianas empresas restauranteras en México: un abordaje comparativo

\section{Materiales y método}

La investigación es de tipo descriptiva transversal realizada en el primer semestre del año 2019, corresponde al trabajo de campo en dos restaurantes por medio de una encuesta dirigida a los comensales; el cuestionario incluye las cuatro dimensiones de la mercadotecnia de servicios, así como las subdimensiones e indicadores seleccionados. El instrumento se basa en la propuesta de Villanueva Cueva (2017) dentro de la línea de investigación Gestión de empresas turísticas y centros de esparcimiento. De esta forma, se limita el cuestionario original de 27 ítems a un total de 20, ya que se eliminaron las preguntas que no eran específicas para un restaurante de comida regional, con un apartado adicional de datos demográficos; las preguntas son de respuesta múltiple.

El cuestionario de Villanueva Cueva (2017) contiene 27 ítems, fue utilizado en cuatro restaurantes del Centro Histórico de la ciudad de Trujillo, Perú; realizó una investigación de tipo exploratorio descriptivo con diseño transversal, la finalidad fue determinar las $4 \mathrm{C}$ del Marketing de servicio en los restaurantes de 3 a 5 tenedores (de lujo, primera clase y segunda clase). La muestra para una población infinita se eligió bajo dos criterios (personas que se encuentren para consumo en el restaurante o que hayan visitado el establecimiento más de tres oportunidades). La validez se basó en la evaluación por tres expertos.

El cuestionario para su aplicación en los restaurantes de la región de Sinaloa México, toma en cuenta una población infinita y considera como requisito de la muestra que se encuentren y hayan consumido en el restaurante, por lo que se aborda a los clientes una vez que el personal del establecimiento haya retirado el servicio de alimentos. El cuestionario puede ser respondido en un promedio de dos minutos, según la prueba piloto llevada a cabo con 30 personas. La ficha técnica de la tabla1, indica las características de la encuesta.

Tabla1.Ficha técnica de la encuesta a clientes

\begin{tabular}{|c|c|}
\hline Características & Encuesta \\
\hline Unidad de estudio & $\begin{array}{l}\text { Dos restaurantes de comida regional elegidos } \\
\text { por convenienciade acuerdo a su disponibilidad. }\end{array}$ \\
\hline Población & $>100000$ \\
\hline Localización & Municipio de Culiacán Sinaloa, México \\
\hline Tamaño muestral & \\
\hline Trabajo de campo & Primer semestre de 2019 \\
\hline Procedimiento & Autoadministrado entrega en mano \\
\hline
\end{tabular}

Fuente: Elaboración propia.

El cuestionario es autoadministrado por el cliente en el establecimiento al que se le entrega un formulario impreso previamente se integra un equipo de encuestadores a quienes se instruye sobre el procedimiento acordado con el restaurante y las reglas de comportamiento en el diálogo con los clientes. En las posibles limitaciones del trabajo de campo, la más sobresaliente es recibir una respuesta negativa de los clientes para el llenado del cuestionario, a lo que se planea contrarrestar con dos acciones: acceder a mesas con tres o más comensales y solicitar el dato de los días laborales con mayor afluencia a criterio del restaurante. Con los ajustes hechos al cuestionario de Villanueva Cueva (2017) las opciones de respuesta, definidas 
las dimensiones, indicadores y preguntas, se incluyen del cuadro número2 al cuadro número 5.La estrategia metodológica corresponde a una investigación descriptiva transversal, por lo que "supone un corte en el tiempo para analizar, en ese momento concreto, determinados aspectos y sacar conclusiones, sin fundamentar el procedimiento en la búsqueda de relaciones causaefecto" (García Ferrer, 2006, p.45).

Tabla 2. Dimensión del cliente

\begin{tabular}{|c|c|c|c|c|}
\hline Ítem & & Opcion & de respue & \\
\hline $\begin{array}{l}\text { 1. ¿Cuál es su nivel de satisfacción en } \\
\text { cuanto a los platillos? }\end{array}$ & $\begin{array}{l}\text { Muy } \\
\text { positiva }\end{array}$ & Positiva & Indiferente & Negativa \\
\hline $\begin{array}{l}\text { 2. ¿Cuál es su nivel de satisfacción sobre } \\
\text { la atención que le brindan? }\end{array}$ & $\begin{array}{l}\text { Muy } \\
\text { positiva }\end{array}$ & Positiva & Indiferente & Negativa \\
\hline $\begin{array}{l}\text { 3. ¿Cuál es su nivel de satisfacción } \\
\text { acerca de la experiencia en el } \\
\text { restaurante? }\end{array}$ & $\begin{array}{l}\text { Muy } \\
\text { positiva }\end{array}$ & Positiva & Indiferente & Negativa \\
\hline $\begin{array}{l}\text { 4. ¿Qué beneficios adicionales busca del } \\
\text { restaurante? }\end{array}$ & Calidad & Servicio & Economía & Comodidad \\
\hline $\begin{array}{l}\text { 5. ¿Cuál es su frecuencia de visita el } \\
\text { mes? }\end{array}$ & $\begin{array}{l}1 \text { a } 3 \\
\text { veces }\end{array}$ & $\begin{array}{l}\text { Más de } 3 \\
\text { veces }\end{array}$ & & \\
\hline
\end{tabular}

Fuente: Elaboración propia

Tabla 3. Dimensión del costo

\begin{tabular}{|c|c|c|c|c|}
\hline Ítem & \multicolumn{4}{|c|}{ Opciones de respuesta } \\
\hline $\begin{array}{l}6 \text { ¿Cuánto tiempo } \\
\text { permanece en el } \\
\text { restaurante? }\end{array}$ & $\begin{array}{l}1 \text { hora } \\
\text { o menos }\end{array}$ & 1 a 3 horas & $\begin{array}{l}\text { Más de } 3 \\
\text { horas }\end{array}$ & \\
\hline $\begin{array}{l}7 \text { ¿Cuánto es el } \\
\text { promedio que gasta? }\end{array}$ & $\begin{array}{l}100 \text { pesos } \\
\text { o menos }\end{array}$ & 100 a 300 pesos & $\begin{array}{l}\text { Más de } 300 \\
\text { pesos }\end{array}$ & \\
\hline $\begin{array}{l}8 \text { ¿En relación al precio } \\
\text { de los platillos usted } \\
\text { está? }\end{array}$ & $\begin{array}{l}\text { Muy } \\
\text { satisfecho }\end{array}$ & Satisfecho & Indiferente & Insatisfecho \\
\hline $\begin{array}{l}9 ¿ \text { ¿Cuál es la razón } \\
\text { principal por la que } \\
\text { visita el restaurante? }\end{array}$ & Alimentarse & Convivencia social & $\begin{array}{l}\text { Ambiente } \\
\text { cómodo }\end{array}$ & $\begin{array}{l}\text { Mejores } \\
\text { productos y } \\
\text { servicio }\end{array}$ \\
\hline $\begin{array}{l}10 \text { ¿Le agradaría que } \\
\text { el restaurante haga } \\
\text { esto por usted? }\end{array}$ & $\begin{array}{l}\text { Ofrezca } \\
\text { regalos o } \\
\text { recuerdos }\end{array}$ & $\begin{array}{l}\text { Premiar } \\
\text { constante }\end{array}$ & $\begin{array}{l}\text { Informar } \\
\text { sobre } \\
\text { eventos }\end{array}$ & $\begin{array}{l}\text { Promoción de } \\
\text { platillos }\end{array}$ \\
\hline $\begin{array}{l}11 \text { ¿El restaurante le } \\
\text { ha brindado alguna } \\
\text { cortesía? }\end{array}$ & $\mathrm{Si}$ & No & & \\
\hline $\begin{array}{l}\text { 12 ¿Recomendaría el } \\
\text { restaurante entre sus } \\
\text { familiares, amigos y } \\
\text { conocidos? }\end{array}$ & $\mathrm{Si}$ & No & & \\
\hline
\end{tabular}

Fuente: Elaboración propia

Tabla4.Dimensión de conveniencia

\begin{tabular}{cc|lcl}
\hline \multicolumn{4}{c}{ Ítem } & Opciones de respuesta \\
\hline $\begin{array}{l}\text { 13¿Está conforme con la la } \\
\text { ubicación del restaurante? }\end{array}$ & $\begin{array}{l}\text { Muy } \\
\text { Conforme }\end{array}$ & Conforme Indiferente & $\begin{array}{l}\text { No está } \\
\text { conforme }\end{array}$ \\
\hline
\end{tabular}


Indicadores en el servicio en las pequeñas y medianas empresas restauranteras en México: un abordaje comparativo

\begin{tabular}{|c|c|c|c|c|}
\hline $\begin{array}{l}14 ¿ \text { Está dispuesto a visitar } \\
\text { nuevas sucursales en otros } \\
\text { lugares lejanos? }\end{array}$ & $\begin{array}{l}\text { Muy de } \\
\text { Acuerdo }\end{array}$ & $\begin{array}{l}\text { De } \\
\text { acuerdo }\end{array}$ & Indiferente & $\begin{array}{l}\text { En } \\
\text { Desacuerdo }\end{array}$ \\
\hline $\begin{array}{l}15 \text { ¿Qué servicio adicional } \\
\text { prefiere? }\end{array}$ & \multirow{2}{*}{$\begin{array}{l}\text { Estacionamiento } \\
\text { Variedad de } \\
\text { platillos } \\
\end{array}$} & \multirow{2}{*}{$\begin{array}{l}\text { Servicio } \\
\text { para llevar } \\
\text { Bufet }\end{array}$} & \multirow{2}{*}{$\begin{array}{l}\text { Servicio a } \\
\text { domicilio } \\
\text { Especialidad de } \\
\text { la casa }\end{array}$} & \multirow{2}{*}{$\begin{array}{l}\text { Internet } \\
\text { gratis } \\
\text { Platillo } \\
\text { personal }\end{array}$} \\
\hline 16. ¿Qué prefiere usted? & & & & \\
\hline
\end{tabular}

Fuente: Elaboración propia

Tabla5.Dimensión de comunicación

\begin{tabular}{|c|c|c|c|c|}
\hline Ítem & \multicolumn{4}{|c|}{ Opciones de respuesta } \\
\hline $\begin{array}{l}17 \text { ¿De qué forma prefiere hacer } \\
\text { reservación? }\end{array}$ & Teléfono & $\begin{array}{l}\text { Internet o redes } \\
\text { sociales }\end{array}$ & & \\
\hline $\begin{array}{l}\text { 18¿Por qué medios se enteró del } \\
\text { restaurante? }\end{array}$ & $\begin{array}{l}\text { Amigos o } \\
\text { familia }\end{array}$ & $\begin{array}{l}\text { Compañeros de } \\
\text { trabajo }\end{array}$ & $\begin{array}{l}\text { Internet o redes } \\
\text { sociales }\end{array}$ & $\begin{array}{l}\text { Medios } \\
\text { publicitarios }\end{array}$ \\
\hline $\begin{array}{l}19 ¿ \text { Qué tan satisfecho está con el } \\
\text { restaurante en la atención de quejas } 0 \\
\text { sugerencias? }\end{array}$ & $\begin{array}{l}\text { Muy } \\
\text { satisfecho }\end{array}$ & Satisfecho & Indiferente & Insatisfecho \\
\hline $\begin{array}{ll}20 \text { 20 Considera importante los } \\
\text { espectáculos y eventos? }\end{array}$ & $\begin{array}{l}\text { Muy } \\
\text { importante }\end{array}$ & Importante & Indiferente & $\begin{array}{l}\text { No es } \\
\text { importante }\end{array}$ \\
\hline
\end{tabular}

\section{Fuente:Elaboración propia}

El manejo de los datos involucra un proceso, que a recomendación de Ortiz Ocaña(2015, p.37) comprende: "la descripción, registro, análisis e interpretación de la naturaleza actual, y la composición o procesos de los fenómenos". Con la herramienta de formulario Google como medio de administración, para uso del equipo de encuestadores en la captura de la totalidad de cuestionarios, el análisis corresponde a datos de estadística descriptiva.

El análisis de resultados, en el orden delos 20 indicadores de la encuesta, se facilita con la disponibilidad de la frecuencia de respuesta para elaborar gráficas y tablas comparativas que lleven a la interpretación de resultados. Una técnica auxiliar es la observación no participante, esta ayuda a complementar la descripción del establecimiento, iniciada con las fotografías del restaurante; la guía de observación contiene: el menú o carta, accesos, distribución del área y secciones, ornamentación, imagen exterior o publicidad, aspecto personal y rasgos de amabilidad de los empleados.

\section{Resultados}

Los resultados de la encuesta son preliminares, con lo que se busca responder a la pregunta problematizadora: ¿qué opinan los clientes acerca del servicio de restaurante regional, la atención de necesidades, el costo, la conveniencia y la comunicación? Los restaurantes seleccionados corresponden a Pascola y Casa de Peraza que atienden un flujo constante de consumidores de la región y en los que los propietarios apoyaron la aplicación de la encuesta a los clientes al terminar de consumir los alimentos.

Las características observadas de los restaurantes, en primer lugar, Pascola es un negocio considerado dentro de los 10 mejores en su tipo en la localidad. Cuenta con dos sucursales, la más antigua se localiza en el desarrollo urbano Tres Ríos en la ciudad de Culiacán, 
Sinaloa, México. Ofrece comida indígena originaria del norte de Sinaloa. El establecimiento está ambientado con una temática basada en la danza de los Pascolas, por la cual lleva su nombre; uno de los atributos del restaurante son los eventos artísticos y la venta de artesanías, el más característico es la demostración de la danza del venado y la ambientación musical propia del grupo indígena mayo.

Casa de Peraza es un restaurante regional, folclórico y colorido; que se encuentra entre los mejores 15 de comida típica sinaloense ubicado fuera de la ciudad con un ambiente campestre. En las instalaciones predominan los colores amarillo, verde, rosa y azul, ambientado al estilo de pueblo señorial. La decoración es con diversos objetos como flores de colores desde el techo y aves (chuparrosa, también conocida como colibrí). El mobiliario es un comedor rústico de madera al aire libre rodeado de árboles de la región, diseñado para degustar la cocina artesanal y todo está hecho a mano; dispone de un área con aire acondicionado. El ambiente se percibe agradable e informal para reuniones, además ofrece entrada con acceso para silla de ruedas. Se localiza en el municipio de Culiacán Sinaloa, México. Ambos restaurantes se consideran de gran tradición y representativos de la comida local. Con antigüedad en el mercado superior a los 10 años.

Los resultados del análisis comparativo en los dos restaurantes se presentan en la tabla número 6a la tabla número 9 , en el orden de las dimensiones del consumidor, costo, conveniencia y comunicación.

En la dimensión del cliente o consumidor (la primera de las $4 \mathrm{C}$ del modelo de Lauterborn), los resultados de la encuesta son similares; los indicadores de productos, servicios y experiencia se encuentran alrededor de un $60 \%$, los beneficios adicionales que prefieren son la calidad/servicio; y la frecuencia en la que acuden al restaurante es de 1 a 3 veces al mes. Estos resultados coinciden con los principales factores que propone Rodríguez-López et al. (2020) que son determinantes en la experiencia gastronómica que involucra la calidad de los platillos, del servicio y del ambiente físico y esto les ha permitido a Pascola y a Casa de Peraza éxito en su negocio.

Tabla 6. Resultados en la dimensión del consumidor

\begin{tabular}{|c|c|c|c|c|}
\hline \multirow[t]{2}{*}{ Dimensión } & \multirow[t]{2}{*}{ Pregunta } & \multirow[t]{2}{*}{ Indicador } & \multicolumn{2}{|c|}{ Restaurante regional } \\
\hline & & & Pascola & Casa de Peraza \\
\hline \multirow[t]{5}{*}{ Enfoque al cliente } & 1 & Productos & $61.2 \%$ positiva & $64.2 \%$ positiva \\
\hline & 2 & Servicio & $64.2 \%$ positiva & 53.7\% positiva \\
\hline & 3 & Experiencia & $64.2 \%$ positiva & $59.7 \%$ positiva \\
\hline & 4 & $\begin{array}{l}\text { Beneficio } \\
\text { adicional }\end{array}$ & $67.2 \%$ calidad & $67.2 \%$ calidad \\
\hline & 5 & $\begin{array}{l}\text { Frecuencia } \\
\text { mensual }\end{array}$ & $77.6 \% 1-3$ veces & $76.1 \% 1-3$ veces \\
\hline
\end{tabular}

Fuente: Elaboración propia 
Indicadores en el servicio en las pequeñas y medianas empresas restauranteras en México: un abordaje comparativo

En la tabla 7 de resultados en la dimensión del costo-beneficio (la segunda de las $4 \mathrm{C}$ del modelo de Lauterborn), la permanencia de tiempo en el restaurante es de 1 a 3 horas en un $60 \%$ aproximado. El $73 \%$ de clientes de Pascola gasta entre 100 y 300 pesos, comparado con el $50 \%$ de clientes de Casa de Peraza. La satisfacción por los precios supera el $64 \%$ en ambos, la necesidad que cubre el servicio es la convivencia social, la mayoría no recibe cortesías y sugiere que se ofrezcan promociones como la de cliente frecuente. Entre ambos restaurantes el comensal busca la convivencia de acuerdo con el $43 \%$ que arroja el estudio y con respecto a las promociones el $68.7 \%$ es un cliente frecuente mientras que en el restaurante Casa de Peraza solamente el $43.3 \%$. En cortesías hay un alto porcentaje que no las recibe superando más del $50 \%$ en ambos casos, por lo que el restaurante Pascola los entrevistados contestaron que no reciben ningún tipo de cortesía en un $77.6 \%$ y en el restaurante Casa de Peraza 67.2\%Los dos restaurantes son altamente recomendables por los clientes, y el que es más recomendable es el restaurante Pascola con un $100 \%$ mientras que el restaurante Casa de Peraza un $86.6 \%$. Esta dimensión coincide con De Sá (2012) en el aspecto de lo que está dispuesto a pagar el cliente por asistir al restaurante y en el beneficio que obtiene al consumir los platillos y la experiencia lo que refleja su lealtad de una a tres veces por mes logrando un marketing relacional positivo en el presente y en el futuro.

Tabla 7. Resultados en la dimensión del costo

\begin{tabular}{|c|c|l|l|l|}
\hline \multirow{2}{*}{ Dimensión } & Pregunta & \multicolumn{1}{|c|}{ Indicador } & \multicolumn{2}{|c|}{ Restaurante regional } \\
\cline { 4 - 5 } & & \multicolumn{2}{|c|}{ Pascola } & \multicolumn{1}{c|}{ Casa de Peraza } \\
\hline $\begin{array}{c}\text { Enfoque al } \\
\text { costo } \\
\text { beneficio }\end{array}$ & 6 & Tiempo & $\begin{array}{l}64.2 \% \\
1-3 \text { horas }\end{array}$ & $\begin{array}{l}58.2 \% \\
1-3 \text { horas }\end{array}$ \\
\cline { 2 - 5 } & 7 & Gasto & $\begin{array}{l}73.1 \% \\
\$ 100 \text { a } 300\end{array}$ & $\begin{array}{l}50.7 \% \\
\$ 300 \text { o más }\end{array}$ \\
\cline { 2 - 5 } & 8 & Precios & $\begin{array}{l}74.6 \% \\
\text { satisfecho }\end{array}$ & $\begin{array}{l}64.2 \% \\
\text { Satisfecho }\end{array}$ \\
\cline { 2 - 5 } & 9 & Necesidad & $43.3 \%$ convivencia & $\begin{array}{l}43.3 \% \\
\text { Convivencia }\end{array}$ \\
\cline { 2 - 5 } & 15 & Promociones & $\begin{array}{l}68.7 \% \\
\text { cliente frecuente }\end{array}$ & $\begin{array}{l}43.3 \% \\
\text { cliente frecuente }\end{array}$ \\
\cline { 2 - 5 } & 19 & Cortesías & $77.6 \%$ no recibe & $67.2 \%$ no recibe \\
\cline { 2 - 5 } & 20 & Recomendación & $\begin{array}{l}100 \% \\
86.6 \% \\
\text { lo recomienda }\end{array}$ \\
\hline
\end{tabular}

Fuente: Elaboración propia

En la tabla 8 de la dimensión de conveniencia (la tercera de las 4C del modelo de Lauterborn), que se relaciona con la accesibilidad para los comensales, indica que más del $50 \%$ de los clientes están conformes con la ubicación y alrededor del $60 \%$ están de acuerdo en acudir a un nuevo domicilio; el estacionamiento es el servicio adicional preferido en ambos restaurantes coincidiendo con el $41.8 \%$ y en cuanto a la variedad de productos, los clientes de Pascola eligen la variedad de platillos con un $55.2 \%$ y los de Casa de Peraza con el $44.8 \%$ del bufet.

Con los resultados obtenidos en la tabla 8 se aprecia que los dos restaurantes han podido lograr el desarrollo de una lealtad del cliente y de una relación duradera en el presente y 
en el futuro al estar convencidos de poder asistir a la ubicación de una nueva sucursal y esta información coincide con Lauterborn (1990); De Sá (2012). y con Rodríguez-López et al., (2020) en la calidad del producto al ofrecer variedad en el menú y en el servicio porque ofrecen seguridad al contar con estacionamiento y buena ubicación.

Tabla 8. Resultados en la dimensión de conveniencia

\begin{tabular}{|l|c|l|l|l|}
\hline \multicolumn{1}{|c|}{ Dimensión } & Pregunta & \multicolumn{1}{|c|}{ Indicador } & \multicolumn{2}{|c|}{ Restaurante regional } \\
\cline { 3 - 5 } & & & \multicolumn{1}{|c|}{ Pascola } & \multicolumn{1}{|c|}{ Casa de Peraza } \\
\hline \multirow{2}{*}{$\begin{array}{l}\text { Enfoque a la } \\
\text { conveniencia }\end{array}$} & 10 & Ubicación & $59.7 \%$ conforme & $53.7 \%$ conforme \\
\cline { 2 - 5 } & 11 & Nueva ubicación & $67.2 \%$ de acuerdo & $59.7 \%$ de acuerdo \\
\cline { 2 - 5 } & 12 & $\begin{array}{l}\text { Producto o servicio } \\
\text { adicional }\end{array}$ & $41.8 \%$ estacionamiento & $\begin{array}{l}41.8 \% \\
\text { estacionamiento }\end{array}$ \\
\cline { 2 - 5 } & 14 & Variedad de productos & $\begin{array}{l}55.2 \% \text { variedad de } \\
\text { platillos }\end{array}$ & $44.8 \%$ bufet \\
\hline
\end{tabular}

Fuente: Elaboración propia

En la tabla 9 se muestran los resultados de la dimensión de la comunicación (cuarta y última de las $4 \mathrm{C}$ del modelo de Lauterborn), en donde se expone que un $50 \%$ o más de los clientes se apoyan en el uso tradicional del teléfono como medio de contacto con el restaurante, en la misma forma la tradición se conserva en el medio para conocer el establecimiento a través de la familia o amigos; los clientes están satisfechos con las quejas atendidas en un $61.2 \%$ en Pascola y un $44.8 \%$ en Casa de Peraza. Los eventos culturales organizados por el restaurante, los clientes valoran que son importantes en un aproximado del $50 \%$.

También se aprecia que se utiliza al marketing relacional que se basa en establecer relaciones duraderas con los clientes a través de utilizar medios de contacto como lo es el teléfono, el uso de las redes sociales, la familia y el atender las quejas, donde no solamente es importante la venta en el momento, sino que se le da seguimiento a la relación con el cliente y la importancia de generar eventos. Estos resultados de la dimensión de comunicación coinciden con Rodríguez-López et al., (2020) porque ofrecen calidad en el servicio, confianza, capacidad de responder a través de la atención de quejas y empatía al ofrecer eventos culturales.

Tabla 9. Resultados en la dimensión comunicación

\begin{tabular}{|l|c|l|l|l|}
\hline Dimensión & Pregunta & Indicador & \multicolumn{2}{|c|}{ Restaurante regional } \\
\cline { 4 - 5 } & & & \multicolumn{1}{|c|}{ Pascola } & Casa de Peraza \\
\hline $\begin{array}{l}\text { Enfoque a la } \\
\text { comunicación }\end{array}$ & 13 & $\begin{array}{l}\text { Medio de } \\
\text { contacto }\end{array}$ & $\begin{array}{l}50.7 \% \text { internet } \\
\text { y redes sociales }\end{array}$ & $68.7 \%$ por teléfono \\
\cline { 2 - 5 } & 16 & $\begin{array}{l}\text { Medio para } \\
\text { conocerlo }\end{array}$ & $70.1 \%$ familia/amigos & $77.6 \%$ familia/amigos \\
\cline { 2 - 5 } & 17 & $\begin{array}{l}\text { Atención de } \\
\text { quejas }\end{array}$ & $61.2 \%$ satisfecho & $44.8 \%$ satisfecho \\
\hline
\end{tabular}


Indicadores en el servicio en las pequeñas y medianas empresas restauranteras en México: un abordaje comparativo

Tabla 9. Resultados en la dimensión comunicación

\begin{tabular}{|c|c|c|c|c|}
\hline Dimensión & Pregunta & Indicador & \multicolumn{2}{|c|}{ Restaurante regional } \\
\cline { 4 - 5 } & & & Pascola & Casa de Peraza \\
\cline { 3 - 5 } & 18 & $\begin{array}{l}\text { Importancia } \\
\text { de eventos }\end{array}$ & $43.3 \%$ importante & $49.3 \%$ importante \\
\hline
\end{tabular}

Fuente: Elaboración propia

En cuanto a los aspectos demográficos de los respondientes se les preguntó la edad, el género y el estado civil. En el restaurante Pascola en el segmento de los 19-30 años de edad correspondió al $34 \%$ de la muestra; en el segmento de 30 años o más fue el $50.7 \%$ y otro segmento, el $15 \%$. En el restaurante Casa de Peraza, el $53.7 \%$ de la muestra es del segmento de los $19-30$ años; el $35.8 \%$ de 30 años o más y el $7.5 \%$ en otro. El género masculino predominó en ambos restaurantes:55.2\% en el restaurante Pascola y $56.7 \%$ en el restaurante Casa de Peraza. Y por último en el restaurante Pascola el $38.8 \%$ de la muestra que respondió al instrumento son solteros; $50.7 \%$ casados y $10.4 \%$ otro estado civil. En el restaurante Casa de Peraza el $58.2 \%$ de los respondientes son solteros; $37.3 \%$ casados y $4.5 \%$ otro. Se observa que los clientes que asisten al restaurante Pascola son de mayor edad y de estado civil casados, en comparación con los clientes de Casa de Peraza, más jóvenes y solteros.

Respecto a los resultados obtenidos en el aspecto demográfico Londhe (2014) hace referencia que el modelo de las $4 \mathrm{C}$ de Lauterborn ha evolucionado del concepto del marketing Mix conocido como las 4P (Precio, Producto, Plaza y Promoción) con un enfoque de mayor orientación hacia un nicho de mercado donde se consideran aspectos como la edad, sexo, estilos de vida, etc., por lo que en el marketing tradicional o transaccional lo que importa es únicamente el realizar la venta en el momento presente.

Asimismo con la revisión de la literatura en la dimensión del costo que establece Cuevas (2004), Hernández Garnica y Maubert Viveros (2009) con la dimensión de conveniencia y Lerma (2010) en la dimensión de comunicación, se acepta el supuesto debido a que los resultados indican que los consumidores están satisfechos con los precios ya que ofrece un lugar para la convivencia social; están conformes con la ubicación actual y otras ubicaciones proyectadas a futuro; en los servicios adicionales prefieren el estacionamiento y la variedad de platillos o bufet.

\section{Conclusiones}

Para atender la pregunta problematizadora ¿qué opinan los clientes acerca del servicio, la atención de necesidades, costo, conveniencia y comunicación con el restaurante regional? Se formuló como supuesto de la investigación: la opinión es favorable al servicio del restaurante regional con una alta presencia de la comida a base de recetas e ingredientes tradicionales; así mismo los precios y la ubicación son satisfactorios para la experiencia de consumo en el restaurante; por lo tanto se acepta el supuesto planteado con base en los resultados ya que los clientes consideran positivo el nivel de satisfacción en cuanto a los platillos, 
servicio, experiencia y beneficio que reciben de los restaurantes de comida tradicional sinaloense.

Se determina que el servicio de los restaurantes de cocina regional sinaloense presenta una mínima diferencia de los alimentos y bebidas preparados en función de los ingredientes típicos, por lo que la conformación del menú se fortalece con las combinaciones de platillos para el gusto y economía del cliente. Asimismo, desde la valoración de los comensales, la experiencia en el restaurante es positiva en cuanto al precio, ubicación actual, servicios adicionales que reciben, variedad de platillos, principalmente; por to que el servicio es recomendable para la familia y amigos, como un espacio seguro y confortable de convivencia social.

Ambos restaurantes ofrecen alimentos y platillos que son del agrado y del gusto de los clientes, en donde el restaurante Pascola se especializa en ofrecer comida indígena del Norte de Sinaloa misma que es más atractiva para personas de 30 años o más, mientras que en el Restaurant Casa de Peraza su mayor nicho de mercado son personas que tienen entre 19 y 30 años. Ambos establecimientos incluyen clientela que los visita con familias y que recomiendan a los restaurantes en cuanto a calidad, precio, ambiente y variedad de platillos. Otro aspecto relevante es que hay un futuro muy promisorio en ambos restaurantes ya que una gran parte de sus clientes ven con agrado la apertura de nuevas sucursales y por utilizar el teléfono y las redes sociales como herramientas de comunicación para ofrecer sus servicios y los distintos platillos.

La aplicación del Modelo de las 4C de Lauterborn muestra por qué estas dos empresas dedicadas al giro gastronómico son exitosas ya que gran parte de sus planes y estrategias de trabajo tienen el enfoque centrado en el cliente y a través de un programa de atención de quejas, de atender eventos culturales y de tener presencia en las redes sociales y en el teléfono logran la lealtad y las relaciones permanentes en gran parte de sus clientes.

Por último, la casa de Peraza y Pascola como restaurantes de comida tradicional de Sinaloa son una buena alternativa para ir a degustar platillos con una buena y excelente calidad en el servicio lo que los hace ser un lugar con un agradable ambiente familiar y una grata experiencia.

\section{Referencias bibliográficas}

Cuevas, Francisco Javier. (2004). Control de costos y gastos en los restaurantes. Editorial Limusa. México.

De Sá, Jorge. (2012). Las prácticas de gestión de marketing en las cooperativas portuguesas.

Revista de Economía Pública, Social y Cooperativa. No. 76. España (Pp.198-

227).https://www.redalyc.org/articulo.oa?id=17425849009

Díaz Paniagua, Elena y León Sánchez, Miriam. (2014). Gestión administrativa y comercial en restauración. Ediciones Paraninfo. España.

García Ferrer, Gemma. (2006). Investigación comercial (segunda edición). Editorial ESIC. España.

García Ortiz, Francisco; Gil Muela, Mario y García Ortiz, Pedro Pablo.(2011). Operaciones

básicas y servicios en restaurante y eventos especiales. Ediciones Paraninfo. España.

García, Jambel. (2010). Pensamiento Estratégico: herramienta de competitividad para una orientación Gerencial del nuevo milenio. Revista TEACS. Volumen 2, Número 4. 
Indicadores en el servicio en las pequeñas y medianas empresas restauranteras en México: un abordaje comparativo

Venezuela. (Pp. 95-104). http://www.ucla.edu.ve/dac/revistateacs/articulos/Rev4Ens3-Garcia.pdf

González, Jorge; Salazar, Flor; Ortiz, Raúl y Verdugo, Darwin. (2019). Gerencia Estratégica: Herramienta para la toma de decisiones en las organizaciones. Revista TELOS.

Volumen 21. Número 1. Venezuela (Pp.242-267). https://dialnet.unirioja.es/servlet/articulo?codigo $=6773127$

Hernández Garnica, Clotilde y Maubert Viveros, Claudio Alfonso. (2009). Fundamentos de marketing. Editorial Pearson Educación. México.

Hernández Rojas, Ricardo y Dancausa Millán, María Genoveva. (2018). Turismo gastronómico.

La gastronomía tradicional de Córdoba (España). Estudios y Perspectivas en Turismo. Volumen 27, No. 2. España. (Pp.413-430).

INEGI (2017). Anuario Estadístico y Geográfico de Sinaloa. Extraído de https://www.datatur.sectur.gob.mx//TxEF_Docs/SIN_ANUARIO_PDF.pdf

Kotler, Philip y Armstrong, Gary. (2003). Fundamentos de mercadotecnia (sexta edición). Editorial Pearson Educación. México.

Kotler, Philip; Keller, Kevin Lane. (2006). Dirección de marketing (duodécima edición). Editorial

Pearson Educación. México.

Lauterborn, Bob. (1990). New Marketing Litany: Four Ps passé: C-words take over. Advertising Age. Volumen 61, No. 41. Estados Unidos.

http://www.rlauterborn.com/pubs/pdfs/4_Cs.pdf

(Pp. 26).

Lerma Kirchner, Alejandro. (2010). Desarrollo de nuevos productos: una visión general(cuarta edición). Editorial Cengage Learning. México.

Londhe, Bhausaheb. (2014). Marketing Mix for Next Generation Marketing. Procedia

Economics and Finance. Número 11. Estados Unidos (Pp. 335-

340).https://doi.org/10.1016/S2212-5671(14)00201-9

López Alonso, Ana María; Carabias Muñoz, Lorena; Díaz Paniagua, Elena. (2017). Ofertas gastronómicas (segunda edición). Editorial Paraninfo. España.

McCarthy, Jerome. (1960). Basic Marketing: A Managerial Approach (Primera Edición). Irwing. Estados Unidos.

Ortiz Ocaña, Alexander. (2015). Enfoques y métodos de investigación en las ciencias sociales y humanas. Ediciones de la U. Colombia.

Rodríguez-López, María Eugenia, Alcántara-Pilar, Juan Miguel y Rojas-Lamorena, Álvaro José. (2020). La experiencia gastronómica en el restaurante: delimitación teórica y empírica en dos tipos de establecimiento. Cuadernos de Gestión. Volumen 20, Número 1. España (Pp. 181-204). http://doi:10.5295/cdg.180904mr

Tschohl, John y Franzmeier, Steve. (1994). Alcanzando la excelencia mediante el servicio al cliente. Ediciones Díaz de Santos. España.

Villanueva Cueva, Diego Alejandro. (2017). Las 4c del marketing de servicio en los restaurantes de 3 a 5 tenedores del centro histórico de Trujillo. Tesis de pregrado. Facultad de Ciencias Empresariales. Universidad César Vallejo. Perú.

Villegas Becerril, Almudena. (2014). Cocina creativa o de autor. Nuevas tendencias gastronómicas. Ideas propias Editorial. España 